\title{
Targeted treatment of advanced and metastatic breast cancer with lapatinib
}

\author{
Brendan Corkery ${ }^{1,2}$ \\ Norma O'Donovan ${ }^{2}$ \\ John Crown ${ }^{1,2}$ \\ 'St.Vincent's University Hospital, \\ Dublin, Ireland; ${ }^{2}$ National Institute \\ for Cellular Biotechnology, Dublin \\ City University, Dublin, Ireland
}

Correspondence: Brendan Corkery National Institute for Cellular Biotechnology, Dublin City University, Glasnevin, Dublin 9, Ireland

Tel +353 I 7006233

Fax +353 I 7005484

Email brendan.corkery@dcu.ie

\begin{abstract}
Improved molecular understanding of breast cancer in recent years has led to the discovery of important drug targets such as HER-2 and EGFR. Lapatinib is a potent dual inhibitor of HER-2 and EGFR. Preclinical and phase I studies have shown activity with lapatinib in a number of cancers, including breast cancer, and the drug is well tolerated. The main known drug interactions are with paclitaxel and irinotecan. The most significant side-effects of lapatinib are diarrhea and adverse skin events. Rates of cardiotoxicity compare favorably with trastuzumab, a monoclonal antibody against HER-2. This paper focuses on lapatinib in advanced and metastatic breast cancer, which remains an important therapeutic challenge. Phase II and III studies show activity as monotherapy, and in combination with chemotherapy or hormonal agents. Results from these studies suggest that the main benefit from lapatinib is in the HER-2 positive breast cancer population. Combinations of lapatinib and trastuzumab are also being studied and show encouraging results, particularly in trastuzumab-refractory metastatic breast cancer. Lapatinib may have a specific role in treating HER-2 positive CNS metastases. The role of lapatinib as neoadjuvant therapy and in early breast cancer is also being evaluated.
\end{abstract}

Keywords: HER-2, EGFR, erbB, lapatinib, Tykerb $^{\circledR}$, tyrosine kinase

\section{Management issues in treatment of locally advanced and metastatic breast cancer}

The molecular revolution in oncology is having a major impact on the pharmacotherapy of breast cancer, a disease which has long been recognized as the most drug-sensitive of the common adult solid tumors. The majority of patients whose tumors express steroid hormone receptors will respond for variable periods of time to endocrine therapy with varying degrees of clinical benefit. For those whose tumors lack such receptors, and in the case of receptor positive cancers that become resistant to endocrine agents, conventional cytotoxic chemotherapy produces frequent responses.

These responses frequently palliate the distressing symptoms of cancer, and provide a meaningful degree of survival prolongation. The overwhelming majority of these responses end in relapse, however, and cure remains essentially anecdotal. Chemotherapy and endocrine therapy produce a greater impact when they are given to patients with earlier stage disease as "adjuvants" to definitive loco-regional therapy (surgery and or radiotherapy). "Neoadjuvant" or "induction" therapy refers to the strategy of administering systemic treatments to patients prior to definitive loco-regional therapy. Induction therapy can render inoperable tumors resectable, and can increase the prospects for breast conservation. There are also theoretical advantages to the early initiation of systemic therapy in these patients, eg, the delivery of drugs through intact vasculature, in vivo assessment of response to drugs, and the opportunity to study the biological effects of treatment.

Chemotherapy is, however, a very non-specific treatment, which is injurious to normal as well as malignant tissues, producing clinical toxicity, which can be severe. 
In addition, most chemotherapeutic agents were developed empirically, often in advance of an understanding of their mechanisms of action.

Recent years have seen an improved molecular understanding of breast cancer biology, an understanding which has ushered in an era of specific molecular therapeutics for breast cancer. One of the most intensively studied systems, and one which has already proven vulnerable to pharmacotherapy, is the human epidermal growth factor system.

\section{The epidermal growth factor receptor system in breast cancer}

The c-erbB or HER (human epidermal growth factor receptor) family of transmembrane tyrosine kinases consists of four known members (epidermal growth factor receptor (EGFR), HER-2, HER-3, and HER-4). These receptors consist of an extracellular domain, a transmembrane region, and an intracellular domain with tyrosine kinase function except HER-3 which lacks kinase activity. When a ligand binds to a receptor, the receptor forms homo- or hetero-dimers, which leads to the activation of tyrosine residues in the intracellular domain. There are numerous Erb ligands, including epidermal growth factor (EGF), TGF- $\alpha$, epiregulin, amphiregulin, and neuregulins. However, HER-2 has no known ligand, and appears to mediate its effects through dimerization with other members of the HER family. Important downstream proteins activated by these pathways include the PI3K-Akt and the RAF-MEK-MAPK pathways, which have key roles in cell proliferation and survival (reviewed in [Yarden and Sliwkowski 2001]).

The HER-2 gene is over-expressed and/or amplified in approximately $20 \%-30 \%$ of invasive breast cancers and is associated with more aggressive tumor behavior and decreased overall survival (Slamon et al 1987). A review of 40 different studies, which included 5,232 patients, reported that on average $45 \%$ of breast cancers were positive for EGFR (range 14\%-91\%) (Klijn et al 1992). The range of positivity reported may relate to different methods of detecting EGFR. EGFR expression has been associated with poorer prognosis in breast cancer (Sainsbury et al 1987; Toi et al 1994).

Trastuzumab, a monoclonal antibody against HER-2 was the first targeted therapy available in HER-2 over-expressing breast cancer, and has become first-line treatment in both early and advanced disease (Engel and Kaklamani 2007). Trastuzumab acts by binding to the extracellular domain of HER-2 and also mediates antibody-dependent cellular cytoxicity (ADCC) (Cooley et al 1999). Trastuzumab enhances response to chemotherapy and has significantly improved outcomes in this subgroup of patients. However, not all patients with HER-2 over-expressing breast cancers respond to trastuzumab therapy. In the metastatic setting, the response rate to trastuzumab monotherapy is less than $35 \%$ (Vogel et al 2002), and most patients who respond initially develop resistance within two years (Nahta and Esteva 2007). Trastuzumab therapy has also been associated with significant rates of cardiomyopathy, in particular when administered in combination with anthracyclines, or to patients with prior anthracycline exposure. The incidence of brain metastases in patients with HER-2 positive disease increases on trastuzumab treatment, possibly reflecting improved extra-cerebral disease control with this agent which does not cross the blood-brain barrier (BBB) (Clayton et al 2004).

A number of EGFR inhibitors are also licensed for use in clinical oncology. These include the small molecule tyrosine kinase inhibitors gefitinib (Baselga and Averbuch 2000) and erlotinib (Pollack et al 1999), and the monoclonal antibody cetuximab (Goldstein et al 1995). However, to date, clinical trials with EGFR inhibitors have yielded disappointing results in breast cancer therapy. Gefitinib monotherapy in metastatic breast cancer showed response rates of 2\%-13\% (von Minckwitz et al 2005). Erlotinib and cetuximab have been studied in combination with chemotherapy in advanced breast cancer. Reports from trials to date have not shown any enhanced benefit with addition of EGFR inhibitors to chemotherapy. However, a number of trials with these agents are ongoing, and may have therapeutic benefits in specific subgroups of breast cancer, such as triple negative disease.

Lapatinib is a dual inhibitor of HER-2 and EGFR. It has shown promising results in clinical trials in breast cancer and is now approved for the treatment of trastuzumabrefractory HER-2 positive metastatic breast cancer. This review focuses on the use of lapatinib in advanced and metastatic breast cancer.

\section{Lapatinib: pharmacology, mode of action and pharmacokinetics Pharmacology and mode of action}

Lapatinib (GW572016, Tykerb ${ }^{\circledR} /$ Tyverb $^{\circledR}$ ) is an orally bio-available 6-thiazolylquinazoline which is a potent dual inhibitor of EGFR and HER-2 (Gaul et al 2003). Lapatinib binds to the adenosine triphosphate (ATP) binding site of both EGFR and HER-2. Lapatinib binds to the inactive form of EGFR and has a slower dissociation rate than selective EGFR inhibitors, such as erlotinib, which binds to the active form of EGFR. The estimated dissociation constants $\left(\mathrm{K}_{\mathrm{i}}^{\mathrm{app}}\right)$ of 
lapatinib for EGFR and HER-2 are $3.0 \pm 0.2 \mathrm{nM}$ and $13 \pm 1 \mathrm{nM}$ respectively (Wood et al 2004). Lapatinib inhibits purified EGFR and HER-2 with a $>300$-fold selectivity in comparison to other kinases, and with $\mathrm{IC}_{50}$ values $<12 \mathrm{nM}$ (Table 1) (Rusnak et al 2001).

Preclinical models have shown that tumor cell lines (derived from a number of different tumor types including breast, head and neck, vulva, lung, and gastric) which over-express either EGFR or HER-2 are more sensitive to lapatinib. Rusnak et al (2007) described a model for determining lapatinib sensitivity in cell lines. Using a panel of human normal and tumor cell lines (including breast, colon, gastric, head and neck, lung, ovarian, and pancreatic cancer cell lines), $\mathrm{IC}_{50}$ values ranged from $0.025 \mu \mathrm{M}$ to $11.5 \mu \mathrm{M}$ (breast tumors range $0.025-7.01 \mu \mathrm{M}$ ). Cell line sensitivity correlated with receptor function, particularly HER-2, although cell lines with increased EGFR expression and modest HER-2 expression also showed sensitivity. Simultaneous consideration of both receptors was found to be the best predictor of response.

Lapatinib binding of EGFR and HER-2 has been shown to decrease EGFR, HER-2, Raf, AKT, and MAPK activity (Xia et al 2002; Konecny et al 2006; Zhou et al 2006; Nahta et al 2007). MAPK and AKT may be important biomarkers of response to lapatinib. MAPK is involved in proliferation, and AKT is a key pathway in cell survival (Xia et al 2002). Xia et al (2002) reported that lapatinib markedly decreases phosphorylation of MAPK and AKT in the HER-2 overexpressing BT474 breast cancer cell line, and in human tumor xenografts. In vitro, this corresponded with a 23 -fold increase in apoptosis. Using gene expression profiles, Hegde et al (2007) have shown that lapatinib treatment results in strong differential regulation of genes in the Akt pathway in sensitive breast cancer cell lines, compared with less sensitive cell lines (Hegde et al 2007). These include genes involved in cell cycle control and cellular metabolic processes. In particular, lapatinib up-regulates the pro-apoptotic gene FoxO3A in the sensitive breast cancer cell lines BT474 and SKBR3.

Table I Kinase inhibition by lapatinib (GW5720I6) (adapted from Rusnak et al 200I)

\begin{tabular}{ll}
\hline Kinase & IC $_{50}$ with lapatinib (nM) \\
\hline EGFR & $10.8 \pm 0.53$ \\
HER2 & $9.2 \pm 0.75$ \\
HER4 & $367 \pm 4.2$ \\
c-src & $3500 \pm 650$ \\
c-RAF, MEK, ERK,VEGFR-2 & all $>10,000$ \\
\hline
\end{tabular}

Gene expression profiling also showed that lapatinib treatment stimulated hormone receptor expression in cell lines with moderate estrogen and progesterone levels (Hegde et al 2007). Interestingly, lapatinib has been shown to restore tamoxifen sensitivity in a resistant breast cancer cell line, via inhibition of estrogen-stimulated transcriptional activity (Chu et al 2005). Thus, lapatinib may sensitize hormone resistant tumors to hormonal agents.

\section{Pharmacokinetics}

In six healthy volunteers, administered a single oral dose of $250 \mathrm{mg}$ lapatinib, the predominant route of excretion of lapatinib and its metabolites was feces (median $92 \%$, range $60 \%-98 \%$ ). Approximately $20 \%$ of the drug was excreted un-metabolized. Less than $2 \%$ excretion was urinary (EGF10019) (GlaxoSmithKline). Pharmacokinetic studies of single and multiple doses of lapatinib in healthy subjects have shown a lag in detecting serum levels of drug post ingestion, suggesting a delay in oral absorption. Peak serum concentrations occur at a median of three hours (range 1.5-6 hours) (Bence et al 2005). Lapatinib solubility is $\mathrm{pH}$ dependent, and decreases with increasing $\mathrm{pH}$. This suggests that absorption may decrease as the drug passes through the gastro-intestinal (GI) tract (Bence et al 2005). It is recommended that lapatinib should be taken at least 1 hour before or after a meal. In general, increasing dose led to increasing serum levels. A drug half-life of 24 hours has been suggested, which lends itself to a once-daily dosing regime (Bence et al 2005).

Lapatinib exhibited a similar pharmacokinetic profile in heavily pretreated metastatic cancer patients. In the phase I study EGF10004, Burris et al (2005) reported that peak serum concentrations were achieved at 3 to 6 hours. In general, increased dose led to increased serum concentration. The best dose-response activity with lapatinib was found at $1200 \mathrm{mg}$ once daily, while doses from 500 to $1600 \mathrm{mg}$ once daily were well tolerated. Trough serum levels in patients with tumor response to drug ranged from 0.3 to $0.6 \mu \mathrm{g} / \mathrm{mL}$ (Burris et al 2005). Of note, animal studies have suggested drug penetration of tumors can be up to five times that of serum (Clark et al 2002).

\section{Potential drug interactions}

Lapatinib undergoes metabolism by cytochrome P450 isoenzymes. In vitro studies indicate that lapatinib is metabolized predominantly by CYP3A4 and CYP3A5, and to lesser extent by CYP2C19 (Herendeen et al 2004; Smith et al 2004). Healthy volunteers given the CYP3A4 inhibitor 
ketoconozole for 7 days (EGF10013) showed an increase in serum plasma concentration and the elimination half life of lapatinib (Smith et al 2004). Volunteers administered the CYP3A4 inducer carbamazepine (EGF10018) showed decreased lapatinib serum concentrations (Herendeen et al 2004). These data suggest the important role of CYP3A enzymes as one of the main pathways of lapatinib metabolism in vivo. As a result, co-prescribing of any drugs which inhibit or induce CYP3A should be considered carefully.

In the pharmacokinetic analysis of the phase I study combining lapatinib and paclitaxel (EGF10009), paclitaxel increased the area under the plasma concentration-time curve (AUC) of lapatinib by a factor of 1.2 (1.0-1.4), and the maximum plasma concentration $\left(\mathrm{C}_{\max }\right)$ by $1.4(1.1-1.7)$ (Jones et al 2004, GlaxoSmithKline). The AUC of paclitaxel was increased by $1.2(1.1-1.4)$ by the combination. Paclitaxel had been reported as a CYP3A4 substrate (Beulz-Riche et al 2002). These pharmacokinetic data suggest that paclitaxel may act as an inhibitor of CYP3A4.

There was also a significant drug interaction between lapatinib and SN-38, the active metabolite of irinotecan, in a phase I study of lapatinib and FOLFIRI chemotherapy (EGF10011) (Midgley et al 2007). The AUC for SN-38 increased by $41 \%$ and $\mathrm{C}_{\max }$ increased by $32 \%$. No significant pharmacokinetic interactions have been found between lapatinib and capecitabine (Chu et al 2007), trastuzumab (Storniolo et al 2008), letrozole (GlaxoSmithKline), oxaliplatin and 5-FU (Siegel-Lakhai et al 2007), or docetaxel (GlaxoSmithKline).

Membrane-bound drug transporters (eg, ABC efflux transporters P-gp and BCRP) play an important role in the transport of many substrates both into and out of cells, and play a role in tumor resistance to many traditional chemotherapy drugs (O'Connor et al 2007). At physiological levels, lapatinib has been shown to be a both a substrate and an inhibitor of P-gp and BCRP, and an inhibitor of hepatic uptake transporter OATP 1B1 (Polli et al 2008). Interestingly, efflux pumps in the BBB appear to account for low central nervous system (CNS) lapatinib concentrations in health. However, promising responses of brain metastases in clinical studies have led to the suggestion that there may be disruption of efflux pumps in disease (Polli et al 2008).

\section{Safety and tolerability of lapatinib}

The safety and side-effect profile of lapatinib has been extensively monitored (Table 2). The most common toxicities associated with lapatinib use are diarrhea and rash. The potential cardiac toxicity of HER-2 inhibition has also been monitored.

\section{Diarrhea}

Diarrhea is the most commonly reported adverse event in clinical trials with lapatinib. Gastro-intestinal disturbance is a recognized side-effect of EGFR inhibitors. A pooled analysis of diarrhea events in patients treated with lapatinib (1000-1500 mg/day) in 11 completed clinical trials in a variety of solid tumors (phase I/II/III; $n=2093$ ) has recently been published (Crown et al 2008). The drug was administered as monotherapy $(n=926)$ or in combination with capecitabine $(n=198)$ or taxanes $(n=687)$. Overall, diarrhea was reported in $55 \%$ of patients treated with lapatinib, compared to $24 \%$ of those not receiving the drug. Diarrhea occurred more frequently in those treated with lapatinib and capecitabine (65\%), compared with $51 \%$ of patients receiving lapatinib as monotherapy, and 48\% in those receiving lapatinib and a taxane. Most events were grade 1/2. Grade 3 diarrhea occurred in less than $10 \%$ of cases, and grade 4 events were rare $(\leq 1 \%)$. However, fatalities from septic shock have been reported in this setting.

The mechanism of GI upset related to EGFR inhibition has not been fully elucidated, and the side-effect profile of diarrhea with lapatinib use is similar to that reported with other EGFR inhibitors. However, it has been noted from pharmacokinetic studies that the frequency of diarrhea was dose-related, and not related to serum levels of the drug, suggesting a direct local action on the epithelium of the GI tract as a possible mechanism of action (Burris et al 2005; Crown et al 2008).

In general, lapatinib-related diarrheal events were mild to moderate, of early onset, and of short duration. The recommended practical management of diarrhea is not evidence based, but includes accepted steps similar to management of this condition when induced by other drugs (Moy and Goss 2007). Improved understanding of the side-effect potential of lapatinib and early introduction of pro-active measures in clinical studies to reduce this complication have decreased the frequency and severity of GI disturbances.

\section{Cardiac toxicity}

Cardiac toxicity has emerged as one the main adverse events associated with trastuzumab treatment. In the metastatic breast cancer setting, trastuzumab treatment is associated with approximately $10 \%$ grade 3 cardiac toxicity (Slamon et al 2001). Anthracyclines (which are also cardiotoxic) in combination with trastuzumab result in increased cardiotoxicity. As a result, concomitant administration of trastuzumab with anthracyclines is generally not recommended outside clinical trials. It has been noted that trastuzumab-related heart 
Table 2 Phase I studies with lapatinib (GW572016)

\begin{tabular}{|c|c|c|c|c|c|c|}
\hline Study ID (reference) & Setting & MTD/OTR & Cardiac & Diarrhea & Rash & Other \\
\hline EGFI000I (Bence et al 2005) & Healthy & 250 mg (single dose) & NR & $4 \%$ & $8 \%$ & Headache $(23 \%)$ \\
\hline \multirow[t]{2}{*}{ EGFI0003 (Pandite et al 2004) } & Solid tumors & $1800 \mathrm{mg}$ OD & NR & $17 \%$ & $9 \%$ & Nausea (I6\%) \\
\hline & & 900 mg BD & NR & $29 \%$ & $11 \%$ & Nausea (II\%) \\
\hline EGFI0004 (Burris et al 2005) & Metastatic & $500-1600$ mg OD & $1.5 \%$ & $42 \%$ & $31 \%$ & $\begin{array}{l}\text { Fatigue (10\%); } \\
\text { Nausea (13\%) }\end{array}$ \\
\hline EGFI0005 (Chu et al 2007) & Solid tumors & $1250 \mathrm{mg}$ OD + C $2000 \mathrm{mg} / \mathrm{m}^{2} / \mathrm{d}$ & NR & $67 \%$ & $44 \%$ & Nausea (49\%) \\
\hline EGFI0009 (GlaxoSmithKline) & Solid tumors & $1500 \mathrm{mg}$ OD + P $175 \mathrm{mg} / \mathrm{m}^{2} \mathrm{q} 3 \mathrm{wk}$ & $2 \%$ b & $80 \%$ b & $73 \%$ b & $\begin{array}{l}\text { Fatigue }(75 \%)^{\mathrm{b}} \\
\text { Nausea }(73 \%)^{\mathrm{b}}\end{array}$ \\
\hline EGFI002I (GlaxoSmithKline) & Solid tumors & $1250 \mathrm{mg}$ OD + D $75 \mathrm{mg} / \mathrm{m}^{2} \mathrm{q} 3 \mathrm{wk}$ & $2 \%{ }^{c}$ & $71 \%$ & $54 \%$ & Nausea (3I\%) \\
\hline EGFI0023 (Storniolo et al 2008) & $\mathrm{HER} 2+\mathrm{ABC}$ & $1000 \mathrm{mg}$ OD + trastuzumab & $1.9 \%^{\mathrm{d}}$ & $\sim 90 \%{ }^{\mathrm{e}}$ & $\sim 40 \%$ & Fatigue $(52 \%)$ \\
\hline EGFI0030 (GlaxoSmithKline) & $\mathrm{ER}+/ \mathrm{PR}+\mathrm{ABC}$ & $1500 \mathrm{mg}$ OD + letrozole $2.5 \mathrm{mg}$ & $3 \%$ & $77 \%$ & $64 \%$ & Nausea (49\%) \\
\hline
\end{tabular}

NR: not reported; ${ }^{a}$ one patient with asymptomatic LVEF decrease; ${ }^{b}$ adverse side-effects reported are in the group treated with paclitaxel q3wk + lapatinib only; ${ }^{c}$ one patient with congestive cardiac failure; 'one patient with greater than $20 \%$ decrease in left ventricular ejection fraction, which subsequently resolved; estimate from published bar graph; Abbreviations: MTD, maximum tolerated dose; OTD, optimum tolerated dose; OD, once daily; BD, twice daily; C, capecitabine; P, paclitaxel; $D$, docetaxel; ABC, advanced breast cancer; ER, estrogen receptor; PR, progesterone receptor; LVEF, left ventricular ejection fraction.

failure tends to improve with discontinuation of treatment (Ewer et al 2005; Guarneri et al 2006). In contrast, the rate of cardiac toxicity with lapatinib seems to be much lower. No significant cardiac toxicity was reported in the phase I pharmacokinetic study EGF10004 (Burris et al 2005). Perez et al (2008) analyzed cardiac toxicity in 44 lapatinib trials, involving 3689 patients. Lapatinib was administered as monotherapy or in combination with chemotherapy in these studies. Overall, 60 patients (2\%) had a cardiac event, of which 7 were symptomatic. The mean time of onset of these events was $13 \pm 9$ weeks (95\% CI, 2-54 weeks). The mean decrease in left ventricular ejection fraction (LVEF) was $18.8 \% \pm 5.2 \%$ (95\% CI, 11\%-32\%). Fifty-eight percent of the patients had a full $(n=19)$ or partial $(n=16)$ recovery. No predictive factors of LVEF decrease were identified. Many of the patients studied had prior or concurrent treatment including other cardiotoxic drugs such as anthracyclines and trastuzumab. However, the overall rate of cardiotoxicity reported is low and compares favorably with rates reported with trastuzumab (3\%-27\%) (Seidman et al 2002). In relation to the safety of combined lapatinib and trastuzumab, preliminary safety data from a phase IIb study of pre-operative lapatinib combined with trastuzumab and chemotherapy has recently been reported (Guarneri et al 2008). No significant cardiac toxicities have been reported after 24 weeks in the first 20 evaluable patients.

The precise reasons for the higher rate of cardiotoxicity with trastuzumab compared to lapatinib are not entirely clear. Inhibition of HER-2 signaling by trastuzumab may cause cardiomyocyte dysfunction (Grazette et al 2004); however, this does not appear to be the case with lapatinib
(Geyer et al 2006). Although lapatinib and trastuzumab both target HER-2, it has been proposed that these drugs have different mechanisms of action. Spector et al (2007) have shown that GW2974 (a dual EGFR/HER-2 small molecule inhibitor which is similar to lapatinib) initiates a stress response via AMP-activated protein kinase which protects against TNF $\alpha$-mediated cell death in human cardiomyocytes. In contrast, trastuzumab does not activate this key metabolic pathway, and is not cardio-protective in the same way (Spector et al 2007). A proposed differential mechanism of action of trastuzumab relates to the key signaling protein BCL-antagonist of death (BAD). Inhibition of HER-2 reverses BAD inhibition, which plays a role in mitochondrial function and apoptosis in breast cancer. In normal cardiomyocytes, HER-2 antibody-mediated modulation of BAD (which alters the ratio of BCL- $\mathrm{X}_{\mathrm{S}}$ (proapoptotic): $\mathrm{BCl}-\mathrm{X}_{\mathrm{L}}$ (anti-apoptotic)), may cause mitochondrial depolarization, depletion of ATP and contractile dysfunction (Grazette et al 2004). It has been suggested that this is an unique response to antibody binding and may explain the increased cardiotoxicity associated with trastuzumab (Force et al 2007). Monitoring of potential cardiotoxicity is ongoing in trials of lapatinib.

\section{Rash}

Skin rash is a common adverse event associated with lapatinib use. This appears to be a class effect of drugs which inhibit EGFR, including gefitinib and erlotinib, and may be mediated by EGFR inhibition in the epidermis (Gordon et al 2005; Cersosimo 2006; Geyer et al 2006; Lacouture 2006). A recent review analyzed adverse skin events in 1419 patients 
from 8 lapatinib trials (Sweetman et al 2007). These trials included lapatinib monotherapy $(\mathrm{n}=928)$, and lapatinib in combination with capecitabine or paclitaxel $(n=491)$. Fifty-two percent of patients treated with lapatinib, and $73 \%$ of patients with combination treatment experienced adverse skin events. However, most events were low grade (53\% grade 1 or $2 ; 6 \%$ grade 3 ; none grade 4 ) and treatment discontinuation was required in $1 \%$ of cases. Most lapatinib related events occurred early (within 14 days), and were distributed mainly on the trunk, and less frequently on the face (Sweetman et al 2007).

Interestingly, the severity of cutaneous side-effects with other EGFR inhibitors has been associated with improved tumor response (Perez-Soler and Saltz 2005; Moy and Goss 2007). While this has not been fully explored in relation to these drugs, the same may hold true for lapatinib, and may be a source of some comfort to those who experience rash as result of lapatinib use.

\section{Other adverse events associated with lapatinib}

Abnormal liver biochemistry and hyperbilirubinemia events have been reported in a number of lapatinib clinical studies (Bence et al 2005; Chu et al 2007; Siegel-Lakhai et al 2007). An incidence of $0.4 \%$ hepatotoxicity in the entire lapatinib clinical trials program has been reported (http:/www.gsk. com/media/pressreleases/2008/2008_pressrelease_10019. $\mathrm{htm})$. These have been predominantly elevations in liver enzymes. In most cases patients have recovered once lapatinib has been discontinued. One case of hepatic failure related to lapatinib was reported in EGF20009 $(n=138)$, a first-line monotherapy phase II study (Gomez et al 2008). GlaxoSmithKline has recommended increased frequency of serum liver biochemistry monitoring.

Clinical studies have reported cases of interstitial pneumonitis with other EGFR inhibitors including gefitinib (Konishi et al 2005; Sunaga et al 2007). No interstitial pneumonitis occurred in the lapatinib phase I study (EGF10004). There was one case of pulmonary metastases where fatal interstitial pneumonia developed, which was determined to be related to the underlying disease (Burris et al 2005). There was also one case of pneumonitis $(1 / 52)$ with the safety and tolerability study of lapatinib and docetaxel (EGF10021). This occurred at the highest dose used (lapatinib $1500 \mathrm{mg} /$ docetaxel $75 \mathrm{mg} / \mathrm{m}^{2} \mathrm{q} 3 \mathrm{wk}$ ) (GlaxoSmithKline). The overall incidence of pneumonitis with lapatinib treatment appears to be very low.

\section{Quality of life issues}

In general, lapatinib is a well tolerated drug in monotherapy (Burris et al 2005). The most noteworthy side-effects are those listed above, and are generally of low grade.

In the combination study of lapatinib and FOLFOX4 chemotherapy (EGF10010), 10 of the 34 patients went off study as a result of drug-related adverse events, including diarrhea, fatigue, elevated serum bilirubin, weight loss, decreased LVEF, fatigue, hypersensitivity, and thrombocytopenia (Siegel-Lakhai et al 2007). Diarrhea was a significant problem on this study, and this may have been due in part to the chemotherapy regimen.

Formal quality of life (QoL) assessment has recently been presented in relation to EGF100051, the pivotal phase III trial of combined lapatinib and capecitabine treatment in HER-2 positive metastatic breast cancer (Zhou et al 2008). The study aimed to assess clinically important differences between the group receiving the combination $(n=198)$ and capecitabine alone $(n=201)$. Outcome measures included quality of life measures (including the Functional Assessment of Cancer Therapy-Breast (FACT-B) total, FACT-general (FACT-G), trial outcome index (TOI), EQ-5D utility, and EQ-5D visual analogue scale (VAS), and percentage tumor response. Although the result was not statistically significant, a greater proportion of patients receiving the combined treatment achieved a clinically important difference for all QoL questionnaires. Also, there was a positive correlation between higher QoL scores and tumor response in the combination arm, compared to capecitabine alone $(\mathrm{p}=0.02$ to 0.07 ). These data suggest that lapatinib and capecitabine is a regimen that has clinical benefit and a positive impact on patients' quality of life.

\section{Lapatinib efficacy studies Lapatinib as monotherapy}

The efficacy of single agent lapatinib as second-line therapy in advanced/metastatic breast cancer has been studied in a number of trials (Table 3 ). An open-label single arm phase II study in patients with advanced or metastatic HER-2 positive breast cancer that had progressed on prior trastuzumabcontaining regimens (EGF20002) showed an overall response rate of $5.1 \%$ (4/78) (GlaxoSmithKline). A phase II study (EGF20008) examined the safety and efficacy of lapatinib monotherapy in chemotherapy-refractory tumors (Burstein et al 2008). This study included 2 cohorts of patients, HER-2 positive (Cohort A, $\mathrm{n}=140$ ) and HER-2 negative (Cohort B, $n=89$ ). More than $95 \%$ of patients had stage IV disease, and 
Table 3 Phase II trials with lapatinib (GW572016) monotherapy in advanced/metastatic breast cancer

\begin{tabular}{|c|c|c|c|c|}
\hline Study ID (reference) & Lapatinib dose & Population & $\mathbf{n}$ & Response rate \\
\hline EGF20002 (GlaxoSmithKline) & $\begin{array}{l}1250 \mathrm{mg} \text {, amended to } \\
\text { I } 500 \mathrm{mg}\end{array}$ & T-refractory $\mathrm{ABC} / \mathrm{MBC}$ & 78 & $5.1 \%$ \\
\hline EGFI05084a (Lin et al 2008a) & 750 mg BD & $\begin{array}{l}\text { T-refractory/relapsed HER-2+ } \\
\text { with brain metastases }\end{array}$ & 39 & $2.6 \%$ \\
\hline EGFI03009 (Kaufman et al 2008) & $1500 \mathrm{mg}$ OD & $\begin{array}{l}\text { Chemo-/T-refractory/relapsed } \\
\text { IBC, HER-2+ }(n=126) \\
\text { EGFR+/HER-2- }(n=15)\end{array}$ & $|4|$ & $\begin{array}{l}\text { HER-2+: } 40 \% \text { b } \\
\text { EGFR+/HER-2-: I PR }\end{array}$ \\
\hline EGFI00642 (Toi et al 2007) & $1500 \mathrm{mg}$ OD & $\begin{array}{l}\text { Chemo-/T-refractory } \\
\text { LABC/MBC, HER-2+ }(n=45) \text {, } \\
\text { HER-2- }(n=22)\end{array}$ & 67 & $\begin{array}{l}\text { HER-2+: } 24 \% \text { (0 CR, II PR) } \\
\text { HER-2-: } 5 \% \text { (I CR, } 0 \text { PR) }\end{array}$ \\
\hline EGF20008 (Burstein et al 2008) & 1500 mg OD & Chemo-refractory $\mathrm{ABC} / \mathrm{MBC}$ & 229 & HER-2+: $4.3 \%$; HER-2-: $0.0 \%$ \\
\hline EGF20009 (Gomez et al 2008) & $1500 \mathrm{mg}$ OD or $500 \mathrm{mg}$ BD & First-line HER-2+ LABC/MBC & 138 & $24 \%$ \\
\hline
\end{tabular}

${ }^{a}$ An extension arm in EGFI05084 with lapatinib $1250 \mathrm{mg}$ OD + capecitabine $2000 \mathrm{mg} / \mathrm{m}^{2} /$ day is ongoing; bestimated response rate based on preliminary data; cby independent review.

Abbreviations: RR, response rate; ORR, overall response rate; $\mathrm{OD}$, once daily; $\mathrm{BD}$, twice daily; T, trastuzumab; Chemo, chemotherapy; $\mathrm{LABC}$, locally advanced breast cancer; $\mathrm{MBC}$, metastatic breast cancer; IBC, inflammatory breast cancer; PR, partial response; CR, complete response.

nearly all patients had received 3 or more lines of anti-cancer therapy previously. Ninety-seven percent of HER-2 positive patients had received at least 12 weeks of prior trastuzumab therapy. Lapatinib $1500 \mathrm{mg}$ daily was administered, with dose reduction to $1250 \mathrm{mg}$ in the event of grade $3 / 4$ toxicity. The best response was observed in the HER-2 positive cohort. There was an overall response rate of $1.4 \%(0.2 \%-5.1 \%)$ in the HER-2 positive cohort and $0.0 \%(0.0 \%-4.1 \%)$ in the HER-2 negative cohort. The independent review reported that $5.7 \%$ of HER-2 positive patients received a clinical benefit (CB), but there was no $\mathrm{CB}$ in the HER-2 negative group. Median overall survival was 29.4 weeks (cohort A) versus 18.6 weeks (cohort B). These responses were modest, but this was a heavily pretreated cohort. A similar phase II study of lapatinib monotherapy (1500 mg daily) in 67 Japanese patients with refractory positive advanced/metastatic breast cancer (EGF100642) demonstrated a much greater benefit in the HER-2 positive subgroup (response rate $24 \%$ vs $5 \%$ ) (Toi et al 2007). While the trend for benefit in the HER-2 positive cohorts in these studies is the same, the reasons for a greater response rate in the Japanese study are unclear.

A phase II open-label, two stage, study of lapatinib monotherapy in patients with inflammatory breast cancer (IBC) has also been initiated (EGF103009). IBC is a particularly aggressive form of breast cancer, and is associated with a poor prognosis (Chang et al 1998). Initially, patients were recruited in two cohorts of IBC (A) HER-2 positive $(n=32)$ and (B) EGFR positive/HER-2 negative $(n=15)$, which had progressed on prior chemotherapy or trastuzumab. The patients were treated with lapatinib (1500 mg daily) and response was measured by RECIST and skin biopsies. A $50 \%$ response rate was observed in the initial cohort of HER-2 positive patients, and this cohort was expanded to include 126 patients. There was one partial response (PR) among the 15 patients in the EGFR positive/HER-2 negative cohort, and enrolment in this arm was closed (Johnston et al 2008; Kaufman et al 2008). Preliminary analysis of the expanded HER-2 positive arm shows an approximately $40 \%$ response rate, and subgroup analysis of trastuzumabrefractory tumors shows an approximately $50 \%$ response (Kaufman et al 2008). These results suggest that lapatinib monotherapy is active in the treatment of relapsed/refractory HER-2 positive IBC.

A phase II study (EGF20009) assessed the clinical activity and safety of lapatinib as a first line treatment in locally advanced or metastatic HER-2 positive breast cancer without prior HER-2-targeted therapy. The overall response rate was $24 \%(n=138)$ and did not differ significantly between the two dosage groups (1500 mg daily or $500 \mathrm{mg}$ twice daily). The median duration of response was 28.4 weeks, and progression free survival was $63 \%$ at 4 months, and $43 \%$ at 6 months (Gomez et al 2008). This trial suggests a role for first-line lapatinib therapy in locally advanced or metastatic HER-2 positive breast cancer.

It has been noted that patients with trastuzumab-treated HER-2 positive breast cancer tend to have a higher incidence 
of CNS relapse (Bendell et al 2003). This may in part be due to the limited ability of trastuzumab to cross the BBB (Pestalozzi and Brignoli 2000). In EGF20009, 6 patients with stable CNS disease involvement were enrolled. One patient had CNS relapse only, 3 patients had systemic relapse only, 1 patient died before objective assessment, and 1 remained relapse free at the time of discontinuation of treatment. In theory, lapatinib has a greater ability to cross the BBB, although pharmacokinetic studies have shown low CNS levels of the drug in normal healthy animals (Polli et al 2008).

EGF105084 specifically studied the use of lapatinib monotherapy (750 mg twice daily) for brain metastases in HER-2 positive breast cancer with prior trastuzumab therapy $(n=39)$. Preliminary results of this phase II trial have reported an overall CNS response rate of $2.6 \%$ (1 PR), 6 patients with stable disease (15.4\%) and 7 patients with progression free survival (18\%) at 16 weeks (Lin et al 2008a). There were 4 non-CNS PRs. An association between tumor volume reduction, and physician-reported neurological signs and symptoms has also been observed (Lin et al 2008b). An extension arm to EGF105084 for patients with progression of disease allowed the patients to be offered lapatinib $1250 \mathrm{mg}$ daily + capecitabine $2000 \mathrm{mg} / \mathrm{m}^{2} /$ day. Of the 40 patients enrolled to this arm (as of March 2007), $20 \%$ had $\geq 50 \%$ tumor volume reduction (median absolute reduction $6.2 \mathrm{~cm}^{3}$ ), and $40 \%$ had $\geq 20 \%$ reduction (median $3.9 \mathrm{~cm}^{3}$ ) (Lin et al 2007). These early data suggest that the combination of lapatinib and capecitabine may be of benefit in patients whose CNS disease has progressed on monotherapy with lapatinib.

\section{Lapatinib in combination with chemotherapy}

The combination of a lapatinib analogue (GW282974X) with the capecitabine metabolite 5'-deoxy-5-fluorouridine was synergistic in vitro in breast cancer cell lines (Budman et al 2006). A phase I study (EGF10005) found the optimal tolerated regimen for the combination to be $1250 \mathrm{mg}$ lapatinib daily and $2000 \mathrm{mg} / \mathrm{m}^{2} /$ day capecitabine on days 1 to 14 of a 21-day cycle. Four confirmed responses from 45 patients were reported, including 2 breast malignancies ( 1 complete response and 1 partial response) (Chu et al 2007). The complete response occurred in a patient that had disease progression while receiving trastuzumab.

An open-label phase III randomized clinical trial (EGF100151) proceeded on the basis of these encouraging data. A total of 399 patients with locally advanced or metastatic HER-2 positive breast cancer who had progressed after treatments including trastuzumab, anthracyclines, and taxanes were randomized to receive the combination of lapatinib and capecitabine versus capecitabine alone (Table 4). The primary study endpoint was time to disease progression (TTP). An interim analysis was reported in 2006 with 324 patients (Geyer et al 2006), which showed a significantly reduced TTP in the combination arm (median 8.4 months vs 4.4 months in the monotherapy arm; hazard ratio [HR] 0.49; 95\% CI $0.34-0.71 ; \mathrm{p}<0.001)$. A reduction in the number of patients who developed CNS disease in the combination arm in comparison to the monotherapy arm was also reported, although the difference was not significant. As a result of the increased benefit with the combination therapy, accrual was discontinued, and cross-over was offered to those on the monotherapy arm. Seventy-five more patients were included in the updated analyses which have recently been published (Cameron et al 2008). TTP remains highly significant (6.2 months vs 4.3 months; HR 0.57 (95\% CI, 0.43-0.77; $\mathrm{p}<0.001)$. There has been 1 complete response $(\mathrm{CR})(<1 \%)$ in the combination arm, vs $0(0 \%)$ in the monotherapy arm. The odds ratio for overall response was $1.9,(95 \%$ CI, 1.1-3.4, $p=0.017)$. The decreased incidence of CNS metastases with lapatinib therapy was statistically significant in this analysis $(p=0.045)$. These data strongly suggest a benefit for the combination of lapatinib and capecitabine over capecitabine alone in patients with advanced or metastatic HER-2 positive breast cancer that have progressed on other treatments. Lapatinib, combined with capecitabine, has been licensed for use in the US in refractory HER-2 positive metastatic breast cancer on the basis of the EGF100151 data.

Taxane-based chemotherapy has been a mainstay of breast cancer treatment in the adjuvant and metastatic settings for a generation. The efficacy and safety of lapatinib and 3-weekly paclitaxel was established in a phase I study (EGF10009) (Jones et al 2004). The combination of lapatinib and weekly paclitaxel has shown efficacy in the phase II neoadjuvant setting for inflammatory breast cancer (EGF102580), with a response rate of $78.6 \%$ in the HER-2 positive subgroup (GlaxoSmithKline) (Table 4). A phase III, randomized study examined lapatinib combined with paclitaxel as first-line treatment for metastatic breast cancer, which was either HER-2 negative or has never been tested, (EGF30001) (Di Leo et al 2007) (Table 4). In this double-blind study, 579 patients were randomized to receive paclitaxel $\left(175 \mathrm{mg} / \mathrm{m}^{2}\right)$ and either lapatinib (1500 mg daily; $n=293)$ or placebo $(n=286)$. The primary endpoint was to achieve a $40 \%$ increase in median TTP in the intention-to-treat 
population. Patient characteristics were well balanced between both groups. The overall response rate $(35.1 \%$ vs $25.3 \%$ ) was significant (odds ratio [OR] 1.7, $\mathrm{p}=0.008$ ). The clinical benefit rate was $40.5 \%$ versus $31.9 \%(\mathrm{OR}=1.5$, $\mathrm{p}=0.025$ ). There was no significant difference between the two arms in TTP, or overall survival (Di Leo et al 2007). However, significant differences emerged in the HER-2 positive and negative subgroup analyses. Of the samples tested in the combination arm $(n=291), 19 \%$ were HER-2 positive and $74 \%$ were HER-2 negative. The results were similar in the placebo arm $(\mathrm{n}=288)(15 \%$ HER-2 positive vs $77 \%$ HER-2 negative). Importantly, there was a greater response rate in the HER-2 positive subpopulation in the combination arm of $60 \%$ versus $36 \%$ in the placebo arm (OR 2.9, $\mathrm{p}=0.027$ ). This contrasts with a non-significant increased response in the HER-2 negative patients (31\% vs $24 \%, \mathrm{OR}=1.5, \mathrm{p}=0.118$ ). The median duration of response was longer with the combination for the HER-2 positive patients ( 7.4 vs 5.5 months), while the median duration of response was less in the combination arm in HER-2 negative patients compared with paclitaxel alone (6.2 vs 8.5 months). A significant TTP was also seen in HER-2 positive patients (median 8.1 vs 5.8 months, $\mathrm{p}=0.011$ ). The interaction between HER-2 status and the combination treatment was significant by Cox's proportional hazards model $(p=0.032)$. Similar results were found for event free survival. The addition of lapatinib to paclitaxel appears to be of benefit in certain breast cancer subpopulations, eg, HER-2 positive. Analysis is ongoing to refine this observation, eg, correlation of EGFR status and response. Two trials testing weekly paclitaxel and lapatinib in HER-2 positive advanced breast cancer are also underway (EGF104535, EGF105764). These will provide further efficacy data, and explore safety and tolerability issues raised by this study. A large phase III study comparing lapatinib or trastuzumab with taxanes is also planned (EGF108919).

The results of the phase II and this phase III trial, which included both HER-2 positive and HER-2 negative tumors, have shown efficacy of lapatinib in HER-2 positive breast cancer populations but no significant benefit in HER-2 negative patients. As a result the current focus of research for this drug is in HER-2 positive, rather than in HER-2 negative (EGFR positive) breast cancer.

\section{Lapatinib in combination with hormone therapy}

In an in vitro model of acquired resistance to lapatinib, acquired resistance was attributed to a switch in cell survival dependence from HER-2 alone to co-dependence upon ER and HER-2 (Xia et al 2006). Short-term exposure (14 days) to lapatinib also increased ER signaling in breast cancer patients. These findings provided the rationale for developing clinical trials of hormone therapies with lapatinib in HER-2 positive, ER positive breast cancer. There is also evidence that lapatinib can overcome hormone resistance, caused by cross-talk between HER-2 and ER, in preclinical models (Chu et al 2005). A phase III study of lapatinib combined with letrozole versus letrozole alone in post-menopausal women with estrogen-receptor positive metastatic breast cancer is currently ongoing. Patients are randomized to letrozole with or without lapatinib regardless of HER-2 status to test that hypothesis that lapatinib treatment may prevent the conversion of ER positive/HER-2 negative to ER positive/HER-2 positive breast cancer, thus blocking the development of resistance to endocrine therapy (Chowdhury et al 2007). Two phase II trials in hormoneresistant, ER positive metastatic breast cancer are currently examining lapatinib as single agent (NCT00225758) or in combination with tamoxifen (NCT00118157).

\section{Lapatinib as neoadjuvant therapy}

The potential role of lapatinib as neoadjuvant therapy in HER-2 positive locally advanced breast cancer is currently being evaluated. A phase II study reported promising results for the combination of lapatinib and paclitaxel as neoadjuvant therapy in newly diagnosed inflammatory breast cancer (EGF102580). The combined clinical response rate was $78.6 \%$ in the HER-2 positive cohort and $71.4 \%$ in the HER-2 negative/EGFR positive cohort (GlaxoSmithKline). These preliminary data suggest potential activity of the combination in these populations. A phase II study with lapatinib monotherapy is currently recruiting (www.clinicaltrials. gov, NCT00206427) and a phase I/II study of lapatinib and docetaxel with or without combination chemotherapy is underway (EORTC-10054).

\section{Lapatinib in combination with other targeted agents}

The safety and efficacy of lapatinib as monotherapy and in combination with chemotherapy is being recognized. Novel approaches of dual HER-2 targeting, and of combining lapatinib with anti-vascular agents are being investigated.

The combination of lapatinib and trastuzumab enhances apoptosis in HER-2 over-expressing breast cancer cell lines (Xia et al 2005). The optimal tolerated dose of lapatinib and trastuzumab, a monoclonal antibody against HER-2, was 
determined in a phase I study (EGF10023) (Storniolo et al 2008). Interestingly, in this population of heavily pretreated HER-2 positive advanced breast cancers, there was a $16 \%$ overall response rate. Consequently, there has been a phase III trial (EGF104900), comparing the effect of lapatinib (1500 mg) alone to the combination of lapatinib (1000 mg) and trastuzumab, in metastatic HER-2 positive breast cancer which has progressed on prior anthracyclines, taxanes, and trastuzumab therapy $(n=296)$ (Table 4$)$. The combination demonstrated significantly improved progression-free survival (12 weeks vs 8.4 weeks, OR 0.77, p=0.029) and had a similar side-effect profile compared with lapatinib alone. One death was related to cardiac toxicity in the combination arm. Asymptomatic decrease in left ventricular ejection fraction was also more common in the combination arm ( $5 \%$ vs $2 \%$ ). In summary, the data show that combined HER-2 targeting showed better clinical outcomes in comparison with lapatinib alone, in the pretreated metastatic setting, with a similar side-effect profile (O'Shaughnessy et al 2008).

Ongoing trials involving lapatinib and trastuzumab include a phase III trial comparing paclitaxel and trastuzumab with lapatinib or placebo in HER-2 positive metastatic breast cancer (EGF104383), and a phase I study combining lapatinib, trastuzumab, carboplatin and paclitaxel (EGF103892).

Bevacizumab is a monoclonal antibody against VEGF, and has shown promising activity in combination with trastuzumab in preclinical models (Emlet et al 2007). However, the cardiac toxicity profile of this combination has not been established. HER-2 inhibition with lapatinib may be a less cardiotoxic alternative. A phase II single arm trial is evaluating the combination of lapatinib (1500 mg daily) and bevacizumab $(10 \mathrm{mg} / \mathrm{kg}$ iv q2wk) in 50 patients with HER-2 positive metastatic breast cancer. Preliminary data report a 12 week progression-free survival of $62 \%$ in 16 of 21 patients evaluated (3 partial response, 10 stable disease, 3 progressive disease), and the combination is well tolerated (Rugo et al 2008).

A phase II evaluation of lapatinib combined with the anti-angiogenic tyrosine kinase inhibitor pazopanib, versus lapatinib alone (VEG20007) is ongoing in patients who have not received prior treatment for their progressive disease. Preliminary reports show a $44 \%$ versus $30 \%$ response rate for the combination arm, with a $73 \%$ versus $43 \%$ reduction in target lesions at 12 weeks (Slamon et al 2008).

\section{Biomarkers of response to lapatinib}

Similar to trastuzumab treatment, overexpression of HER-2 appears to be the most reliable predictive marker of response to lapatinib in breast cancer. Within the HER-2 positive breast cancer population additional biomarkers will be needed to further define the patients who are likely to benefit from lapatinib treatment.

Biomarkers of response to lapatinib monotherapy were investigated in the phase I study EGF10004 (Spector et al 2005). Although the numbers were limited, clinical response was associated with increased pretreatment expression of HER-2, p-HER-2, Erk1/2, p-Erk1/2, insulin-like growth factor receptor-1, p70 S6 kinase, and transforming growth

Table 4 Completed phase II/III combination trials with lapatinib (GW5720I6) for advanced/metastatic breast cancer

\begin{tabular}{|c|c|c|c|c|c|}
\hline Study ID (reference) & Regimen & Population & Phase & $\mathbf{n}$ & Outcome \\
\hline \multirow[t]{2}{*}{$\begin{array}{l}\text { EGFI02580a } \\
\text { (GlaxoSmithKline) }\end{array}$} & $\begin{array}{l}\mathrm{L} \mid 500 \mathrm{mg} \text { OD } \times 14 \text { days } \\
\rightarrow \mathrm{L} 1500 \mathrm{mg}+\mathrm{P} 80\end{array}$ & $\begin{array}{l}\text { A: HER-2+ } \pm \text { EGFR }+ \\
\text { (IBC) }\end{array}$ & II & 42 & A: RR 78.6\% (95\% Cl, 63.2-89.7) \\
\hline & $\mathrm{mg} / \mathrm{m}^{2} / \mathrm{wk} \times 12$ weeks & B: HER-2-/EGFR + (IBC) & & 7 & B: RR 7I.4\% (95\% Cl, 29.0-96.3) \\
\hline $\begin{array}{l}\text { EGF3000I (Di Leo et al } \\
\text { 2007) }\end{array}$ & $\begin{array}{l}\mathrm{P} I 75 \mathrm{mg} / \mathrm{m}^{2} \mathrm{q} 3 \mathrm{wk}+ \\
\mathrm{L} 1500 \mathrm{mg} \text { OD, or P + } \\
\text { placebo }\end{array}$ & $\begin{array}{l}\text { MBC HER-2- or } \\
\text { untested }\end{array}$ & III & 570 & $\begin{array}{l}\text { TTP: overall no difference, } \\
\text { significant in HER } 2+\text { subpopula- } \\
\text { tion only (see text) }\end{array}$ \\
\hline $\begin{array}{l}\text { EGFI00I5I (Cameron } \\
\text { et al 2008) }\end{array}$ & $\begin{array}{l}\text { L } 1250 \mathrm{mg} \text { OD + } \\
\text { C } 2000 \mathrm{mg} / \mathrm{m}^{2} \text { vs } \\
\text { C } 2500 \mathrm{mg} / \mathrm{m}^{2}\end{array}$ & LABC/MBC HER-2+ & III & 399 & $\begin{array}{l}\text { TTP: HR } 0.57(95 \% \mathrm{Cl}, 0.43-0.77 \text {, } \\
P=0.000 \mathrm{I} 3) \\
\text { OS: HR } 0.78(95 \% \mathrm{Cl}, 0.55-1.12, \\
P=0.177)\end{array}$ \\
\hline $\begin{array}{l}\text { EGFI04900 } \\
\text { (O'Shaughnessy et al } \\
\text { 2008) }\end{array}$ & $\begin{array}{l}\text { L I } 000 \mathrm{mg} \text { OD + T vs } \mathrm{L} \\
\text { I } 500 \mathrm{mg} \text { OD }\end{array}$ & $\begin{array}{l}\text { T-refractory MBC } \\
\text { HER-2+ }\end{array}$ & III & 296 & $\begin{array}{l}\text { PFS: HR } 0.77(95 \% \mathrm{Cl}, 0.6-\mathrm{I} .0 \\
\mathrm{P}=0.029) \\
\text { OS (median): HR } 0.75(95 \% \mathrm{Cl}, \\
0.5-\mathrm{I} . \mathrm{I}, \mathrm{P}=0.106)\end{array}$ \\
\hline
\end{tabular}

${ }^{a}$ neoadjuvant study.

Abbreviations: OD, once daily; RR, response rate;TTP, time to progression; OS, overall survival; PFS, progression-free survival; T, trastuzumab; LABC, locally advanced breast cancer; MBC, metastatic breast cancer; IBC, inflammatory breast cancer; PR, partial response; CR, complete response. 
factor alpha compared with non-responders. Baseline EGFR expression did not correlate with response. HER-2 overexpression predicted response to lapatinib in the monotherapy phase II study EGF20009 (Gomez et al 2007), the phase I lapatinib/capecitabine study EGF100151 (Cameron et al 2008), and the phase III lapatinib/paclitaxel study EGF30001 (Press et al 2008). Press et al (2008) also reported than the benefit from lapatinib in HER-2 positive metastatic breast cancer may be limited to patients with FISH positive or immunohistochemical 3+ staining intensity.

The extracellular domain (ECD) of HER-2 is cleaved by ADAM protease enzymes (Liu et al 2006) and can readily be detected in the serum of HER-2 positive breast cancer patients. After cleavage of the ECD, the remaining p95 form of HER-2 retains kinase activity. This form of HER-2 has been associated with resistance to trastuzumab treatment but is inhibited by lapatinib (Scaltriti et al 2007). Increased pre-treatment levels of serum HER-2 ECD correlate positively with response to lapatinib, and decreasing levels during treatment are associated with clinical benefit in HER-2 positive metastatic breast cancer (Lipton et al 2007). However, response to lapatinib appeared to be independent of baseline HER-2 ECD levels in the pivotal phase III capecitabine/lapatinib trial (EGF100151) (Cameron et al 2008). High baseline levels of HER-2 ECD in HER-2 negative metastatic breast cancer did not predict response to lapatinib and paclitaxel or paclitaxel alone (Finn et al 2008). Neither EGFR expression nor baseline EGFR ECD levels affected progression free survival in the capecitabine/ lapatinib trial (EGF100151) (Cameron et al 2008).

In the phase I monotherapy study EGF10004, inhibition of phosphorylated AKT and MAPK (downstream targets of HER-2 and EGFR) at day 21 were also associated with clinical responses (Spector et al 2005). Loss of PTEN expression (a negative regulator of AKT), which has been associated with resistance to trastuzumab treatment does not appear to preclude response to lapatinib in patients with inflammatory breast cancer (Xia et al 2007). Phosphorylation of p70 S6 kinase, a downstream target of mTOR (mammalian target of rapamycin), has also been reported as a potential biomarker for lapatinib activity in human breast cancer cell lines (Vazquez-Martin et al 2008).

Forkhead box group $\mathrm{O}$ transcriptional factor (FoxO3A) is a downstream target of the PI3 kinase pathway, which regulates cell proliferation, survival, and malignant transformation. FoxO3A has previously been shown as a direct target of EGFR inhibitors, including lapatinib, in two sensitive HER-2 positive breast cancer cell lines, BT474 and
SKBR3 (Krol et al 2007). Gene array data from EGF100151 suggested that elevated baseline FoxO3A mRNA levels and reduced BCL-2 mRNA correlated with response to the combination of lapatinib and capecitabine (Crown et al 2007).

\section{Conclusions}

Although lapatinib is a dual inhibitor of EGFR and HER-2, it is clearly evident from trials including both HER-2 positive and HER-2 negative breast cancer patients, that the benefits of lapatinib treatment are largely restricted to HER-2 positive breast cancer. Lapatinib has shown activity in trastuzumabrefractory HER-2 positive breast cancer and at present is approved, in combination with capecitabine, for treatment of refractory HER-2 positive metastatic breast cancer. Recent trial results suggest that combined trastuzumab and lapatinib treatment show greater efficacy than lapatinib alone in trastuzumab-refractory metastatic breast cancer. Lapatinib has also shown promising activity as first-line treatment for HER-2 positive metastatic breast cancer and the results of ongoing phase III trials will help to define the role of lapatinib in this setting. Lapatinib may also have a specific role for treatment HER-2 positive breast cancer patients with CNS metastases. Due to the apparent lack of cardiotoxicity, lapatinib may also have benefits in combination therapy with anthracyclines. A phase I study of lapatinib with epirubicin is currently underway to determine the safe dose of epirubicin in combination with lapatinib and to examine the cardiotoxicity of this combination. Several large randomized clinical trials are also underway at present to evaluate the benefits of lapatinib treatment for early stage breast cancer.

\section{Disclosures}

JC has received speaker fees and research support from GlaxoSmithKline.

\section{Acknowledgments}

$\mathrm{BC}$ and $\mathrm{JC}$ are funded by a Health Research Board Clinician Scientist Award.

\section{References}

Baselga J, Averbuch SD. 2000. ZD1839 ('Iressa') as an anticancer agent. Drugs, 60(Suppl 1):33-40; discussion 41-2.

Bence AK, Anderson EB, Halepota MA, et al. 2005. Phase I pharmacokinetic studies evaluating single and multiple doses of oral GW572016, a dual EGFR-ErbB2 inhibitor, in healthy subjects. Invest New Drugs, 23:39-49.

Bendell JC, Domchek SM, Burstein HJ, et al. 2003. Central nervous system metastases in women who receive trastuzumab-based therapy for metastatic breast carcinoma. Cancer, 97:2972-7. 
Beulz-Riche D, Robert J, Riche C, et al. 2002. Effects of paclitaxel, cyclophosphamide, ifosfamide, tamoxifen and cyclosporine on the metabolism of methoxymorpholinodoxorubicin in human liver microsomes. Cancer Chemother Pharmacol, 49:274-80.

Budman DR, Soong R, Calabro A, et al. 2006. Identification of potentially useful combinations of epidermal growth factor receptor tyrosine kinase antagonists with conventional cytotoxic agents using median effect analysis. Anticancer Drugs, 17:921-8.

Burris HA 3rd, Hurwitz HI, Dees EC, et al. 2005. Phase I safety, pharmacokinetics, and clinical activity study of lapatinib (GW572016), a reversible dual inhibitor of epidermal growth factor receptor tyrosine kinases, in heavily pretreated patients with metastatic carcinomas. J Clin Oncol, 23:5305-13.

Burstein HJ, Storniolo AM, Franco S, et al. 2008. A phase II study of lapatinib monotherapy in chemotherapy-refractory HER2-positive and HER2-negative advanced or metastatic breast cancer. Ann Oncol, 19:1068-74.

Cameron D, Casey M, Press M, et al. 2008. A phase III randomized comparison of lapatinib plus capecitabine versus capecitabine alone in women with advanced breast cancer that has progressed on trastuzumab: updated efficacy and biomarker analyses. Breast Cancer Res Treat. 10.1007/s10549-007-9885-0.

Cersosimo RJ. 2006. Gefitinib: an adverse effects profile. Expert Opin Drug Saf, 5:469-79.

Chang S, Parker SL, Pham T, et al. 1998. Inflammatory breast carcinoma incidence and survival: the surveillance, epidemiology, and end results program of the National Cancer Institute, 1975-1992. Cancer, $82: 2366-72$

Chowdhury S, Pickering LM, Ellis PA. 2007. Lapatinib: a novel dual tyrosine kinase inihibitor. Targ Oncol, 2:107-12.

Chu I, Blackwell K, Chen S, Slingerland J. 2005. The dual ErbB1/ErbB2 inhibitor, lapatinib (GW572016), cooperates with tamoxifen to inhibit both cell proliferation- and estrogen-dependent gene expression in antiestrogen-resistant breast cancer. Cancer Res, 65:18-25.

Chu QS, Schwartz G, de Bono J, et al. 2007. Phase I and pharmacokinetic study of lapatinib in combination with capecitabine in patients with advanced solid malignancies. $J$ Clin Oncol, 25:3753-8.

Clark KJ, Keith BR, Alligood K, et al. 2002. Pharmacokinetics and pharacodynamics of GW572016 following oral administration to female BT474-bearing CB-17 SCID mice. AAPS PharmSci, 4:W5286.

Clayton AJ, Danson S, Jolly S, et al. 2004. Incidence of cerebral metastases in patients treated with trastuzumab for metastatic breast cancer. $\mathrm{Br} J$ Cancer, 91:639-43.

Cooley S, Burns LJ, Repka T, et al. 1999. Natural killer cell cytotoxicity of breast cancer targets is enhanced by two distinct mechanisms of antibody-dependent cellular cytotoxicity against LFA-3 and HER2/neu. Exp Hematol, 27:1533-41.

Crown J, Cameron D, Martin AM, et al. 2007. Lapatinib (L) plus capecitabine (C) in HER2+ advanced breast cancer (ABC): report of updated efficacy and genearray data. Eur J Cancer Suppl, 5:212.

Crown JP, Burris HA 3rd, Boyle F, et al. 2008. Pooled analysis of diarrhea events in patients with cancer treated with lapatinib. Breast Cancer Res Treat. DOI 10.1007/s10549-007-9860-9.

Di Leo A, Gomez H, Aziz Z, et al. 2007. Lapatinib (L) with paclitaxel compared to paclitaxel as first-line treatment for patients with metastatic breast cancer: A phase III randomised, double-blind study of 580 patients [abstract]. J Clin Oncol, 25S:abstr 1011.

Emlet DR, Brown KA, Kociban DL, et al. 2007. Response to trastuzumab, erlotinib, and bevacizumab, alone and in combination, is correlated with the level of human epidermal growth factor receptor- 2 expression in human breast cancer cell lines. Mol Cancer Ther, 6:2664-74.

Engel RH, Kaklamani VG. 2007. HER2-positive breast cancer: current and future treatment strategies. Drugs, 67:1329-41.

Ewer MS, Vooletich MT, Durand JB, et al. 2005. Reversibility of trastuzumab-related cardiotoxicity: new insights based on clinical course and response to medical treatment. J Clin Oncol, 23:7820-6.
Finn RS, Gagnon R, Di Leo A, et al. 2008. Lapatinib and paclitaxel in HER2-negative, extracellular domain (ECD) positive metastatic breast cancer (MBC) in a randomised phase III study [abstract]. J Clin Oncol, 26(Suppl):abstr 1017.

Force T, Krause DS, Van Etten RA. 2007. Molecular mechanisms of cardiotoxicity of tyrosine kinase inhibition. Nat Rev Cancer, 7:332-44.

Gaul MD, Guo Y, Affleck K, et al. 2003. Discovery and biological evaluation of potent dual ErbB-2/EGFR tyrosine kinase inhibitors: 6-thiazolylquinazolines. Bioorg Med Chem Lett, 13:637-40.

Geyer CE, Forster J, Lindquist D, et al. 2006. Lapatinib plus capecitabine for HER2-positive advanced breast cancer. N Engl J Med, 355:2733-43.

GlaxoSmithKline clinical trial register [online]. Accessed May 272008. URL: http://ctr.gsk.co.uk/Summary/lapatinib/studylist.asp.

Goldstein NI, Prewett M, Zuklys K, et al. 1995. Biological efficacy of a chimeric antibody to the epidermal growth factor receptor in a human tumor xenograft model. Clin Cancer Res, 1:1311-8.

Gomez HL, Chavez MA, Doval DC, et al. 2007. Investigation of tumor biomarkers as response predictors in a monotherapy study with lapatinib (L) as a first line treatment in ErbB2 amplified women with breast cancer [abstract]. J Clin Oncol, 25(18S):abstr 10562.

Gomez HL, Doval DC, Chavez MA, et al. 2008. Efficacy and safety of lapatinib as first-line therapy for ErbB2-amplified locally advanced or metastatic breast cancer. J Clin Oncol, 26:2940-2.

Gordon AN, Finkler N, Edwards RP, et al. 2005. Efficacy and safety of erlotinib $\mathrm{HCl}$, an epidermal growth factor receptor (HER1/EGFR) tyrosine kinase inhibitor, in patients with advanced ovarian carcinoma: results from a phase II multicenter study. Int $J$ Gynecol Cancer, 15:785-92.

Grazette LP, Boecker W, Matsui T, et al. 2004. Inhibition of ErbB2 causes mitochondrial dysfunction in cardiomyocytes: implications for herceptin-induced cardiomyopathy $J$ Am Coll Cardiol, 44:2231-8.

Guarneri V, Frassoldati A, Cagossi K, et al. 2008. CHER LOB Trial: Preoperative chemotherapy plus trastuzumab, lapatinib or both in HER2-positive operable breast cancer - Safety report as per Independent Data Monitoring Committee (IDMC) and preliminary activity data [abstract]. J Clin Oncol, 26(Suppl):abstr 580.

Guarneri V, Lenihan DJ, Valero V, et al. 2006. Long-term cardiac tolerability of trastuzumab in metastatic breast cancer: the M.D. Anderson Cancer Center experience. J Clin Oncol, 24:4107-15.

Hegde PS, Rusnak D, Bertiaux M, et al. 2007. Delineation of molecular mechanisms of sensitivity to lapatinib in breast cancer cell lines using global gene expression profiles. Mol Cancer Ther, 6:1629-40.

Herendeen JM, Smith DA, Stead A, et al. 2004. An open-label, fixed sequence, two period study to evaluate the potential induction of GW572016 metabolism by carbamazepine [abstract]. J Clin Oncol, 22(14S):abstr 3081.

Johnston S, Trudeau M, Kaufman B, et al. 2008. Phase II study of predictive biomarker profiles for response targeting human epidermal growth factor receptor 2 (HER-2) in advanced inflammatory breast cancer with lapatinib monotherapy. J Clin Oncol, 26:1066-72.

Jones SF, Hainsworth JD, Spigel DR, et al. 2004. A phase I study of the dual kinase inhibitor GW572016 in combination with paclitaxel (EGF10009) [abstract]. J Clin Oncol, 22(14S):abstr 2083.

Kaufman B, Trudeau ME, Johnston S, et al. 2008. Clinical activity of lapatinib monotherapy in patients with HER2+ relapsed/refractory inflammatory breast cancer (IBC): Final results of the expanded HER2+ cohort in EGF103009 [abstract]. J Clin Oncol, 26(Suppl): abstr 636 .

Klijn JG, Berns PM, Schmitz PI, et al. 1992. The clinical significance of epidermal growth factor receptor (EGF-R) in human breast cancer: a review on 5232 patients. Endocr Rev, 13:3-17.

Konecny GE, Pegram MD, Venkatesan N, et al. 2006. Activity of the dual kinase inhibitor lapatinib (GW572016) against HER-2-overexpressing and trastuzumab-treated breast cancer cells. Cancer Res, 66:1630-9.

Konishi J, Yamazaki K, Kinoshita I, et al. 2005. Analysis of the response and toxicity to gefitinib of non-small cell lung cancer. Anticancer Res, $25: 435-41$. 
Krol J, Lam E, Coombes C. 2007. Critical role of FoxO3A transciption factor in response to therapy with EGFR inhibitors, gefitinib and lapatinib, in breast cancer [abstract]. J Clin Oncol, 25(18S):abstr 21018.

Lacouture ME. 2006. Mechanisms of cutaneous toxicities to EGFR inhibitors. Nat Rev Cancer, 6:803-12.

Lin N, Paul D, Dieras V, et al. 2007. Lapatinib and capecitabine for the treatment of brain metastases in patients with HER2+ breast cancer - an updated analysis from EGF105084 [abstract]. Breast Cancer Res Treat, 106(S1):abstr 6076.

Lin NU, Carey LA, Liu MC, et al. 2008a. Phase II trial of lapatinib for brain metastases in patients with human epidermal growth factor receptor 2-positive breast cancer. J Clin Oncol, 26:1993-9.

Lin NU, Roché HH, Dieras V, et al. 2008b. A physician-reported neurological signs and symptoms worksheet (NSS WS) in EGF105084: A phase II study of lapatinib (lap) for pts with recurrent brain metastases from HER2+ breast cancer (BC) [abstract]. J Clin Oncol, 26(Suppl): abstr 1078 .

Lipton A, Carney W, Martin A, et al. 2007. ASCO Breast Cancer Symposium 2007. Abstr 105.

Liu, P. C., Liu, X., Li, Y., et al. 2006. Identification of ADAM10 as a major source of HER2 ectodomain sheddase activity in HER2 overexpressing breast cancer cells. Cancer Biol Ther, 5:657-64.

Midgley RS, Kerr DJ, Flaherty KT, et al. 2007. A phase I and pharmacokinetic study of lapatinib in combination with infusional 5-fluorouracil, leucovorin and irinotecan. Ann Oncol, 18:2025-9.

Moy, B, Goss, P. E. 2007. Lapatinib-associated toxicity and practical management recommendations. Oncologist, 12:756-65.

Nahta R, Esteva FJ. 2007. Trastuzumab: triumphs and tribulations. Oncogene, 26:3637-43.

Nahta R, Yuan LX, Du Y, et al. 2007. Lapatinib induces apoptosis in trastuzumab-resistant breast cancer cells: effects on insulin-like growth factor I signaling. Mol Cancer Ther, 6:667-74.

O'Connor R, Clynes M, Dowling P, et al. 2007. Drug resistance in cancer - searching for mechanisms, markers and therapeutic agents. Expert Opin Drug Metab Toxicol, 3:805-17.

O'Shaughnessy J, Blackwell KL, Burstein H, et al. 2008. A randomised study of lapatinib alone or in combination with trastuzumab in heavily pretreated HER $2+$ metastatic breast cancer progressing on trastuzumab therapy [abstract]. J Clin Oncol, 26(Suppl):abstr 1015.

Pandite L, Burris HA, Jones S, et al. 2004. A safety, tolerability, and pharmacokinetic (PK) study of GW572016 in patients with solid tumours [abstract]. J Clin Oncol, 22(14SL):abstr 3179.

Perez EA, Koehler M, Byrne J, et al. 2008. Cardiac safety of lapatinib: pooled analysis of 3689 patients enrolled in clinical trials. Mayo Clin Proc, 83:679-86.

Perez-Soler R, Saltz L. 2005. Cutaneous adverse effects with HER1/ EGFR-targeted agents: is there a silver lining? J Clin Oncol, 23:5235-46.

Pestalozzi BC, Brignoli S. 2000. Trastuzumab in CSF. J Clin Oncol, 18:2349-51.

Pollack VA, Savage DM, Baker DA, et al. 1999. Inhibition of epidermal growth factor receptor-associated tyrosine phosphorylation in human carcinomas with CP-358,774: dynamics of receptor inhibition in situ and antitumor effects in athymic mice. J Pharmacol Exp Ther, 291:739-48.

Polli JW, Humphreys JE, Harmon KA, et al. 2008. The role of efflux and uptake transporters in $\mathrm{N}$-\{3-chloro-4-[(3-fluorobenzyl)oxy]phenyl\}6-[5-(\{[2-(methylsulfonyl)ethyl ]amino $\}$ methyl)-2-furyl]-4quinazolinamine (GW572016, lapatinib) disposition and drug interactions. Drug Metab Dispos, 36:695-701.

Press MF, Finn RS, Di Leo A, et al. 2008. Correlation of HER2 gene amplification, HER2 and EGFR expression (protein and mRNA) with lapatinib efficacy in women with metastatic breast cancer [abstract]. J Clin Oncol, 26(Suppl):abstr 1007.

Rugo HS, Franco S, Munster P, et al. 2008. A phase II evaluation of lapatinib (L) and bevacizumab (B) in HER-2+ metastatic breast cancer (MBC) [abstract]. J Clin Oncol, 26(Suppl):abstr 1042.
Rusnak DW, Alligood KJ, Mullin RJ, et al. 2007. Assessment of epidermal growth factor receptor (EGFR, ErbB1) and HER2 (ErbB2) protein expression levels and response to lapatinib (Tykerb, GW572016) in an expanded panel of human normal and tumour cell lines. Cell Prolif, 40:580-94.

Rusnak DW, Lackey K, Affleck K, et al. 2001. The effects of the novel, reversible epidermal growth factor receptor/ErbB-2 tyrosine kinase inhibitor, GW2016, on the growth of human normal and tumor-derived cell lines in vitro and in vivo. Mol Cancer Ther, 1:85-94.

Sainsbury JR, Farndon JR, Needham GK, et al. 1987. Epidermalgrowth-factor receptor status as predictor of early recurrence of and death from breast cancer. Lancet, 1:1398-402.

Scaltriti M, Rojo F, Ocana A, et al. 2007. Expression of p95HER2, a truncated form of the HER2 receptor, and response to anti-HER2 therapies in breast cancer. $J$ Natl Cancer Inst, 99:628-38.

Seidman A, Hudis C, Pierri MK, et al. 2002. Cardiac dysfunction in the trastuzumab clinical trials experience. J Clin Oncol, 20:1215-21.

Siegel-Lakhai WS, Beijnen JH, Vervenne WL, et al. 2007. Phase I pharmacokinetic study of the safety and tolerability of lapatinib (GW572016) in combination with oxaliplatin/fluorouracil/leucovorin (FOLFOX4) in patients with solid tumors. Clin Cancer Res, 13:4495-502.

Slamon D, Gomez HL, Kabbinavar FF, et al. 2008. Randomised study of pazopanib + lapatinib vs. lapatinib alone in patients with HER2-positive advanced or metastatic breast cancer [abstract]. J Clin Oncol, 26(Suppl): abstr 1016.

Slamon DJ, Clark GM, Wong SG, et al. 1987. Human breast cancer: correlation of relapse and survival with amplification of the HER-2/neu oncogene. Science, 235:177-82.

Slamon DJ, Leyland-Jones B, Shak S, et al. 2001. Use of chemotherapy plus a monoclonal antibody against HER2 for metastatic breast cancer that overexpresses HER2. N Engl J Med, 344,11:783-92.

Smith DA, Bowen C, Herendeen JM, et al. 2004. An open-label, randomized, two-way crossover study to evaluate the potential inhibition of GW572016 metabolism by ketoconazole [abstract]. J Clin Oncol, 22(14S):abstr 3071.

Spector NL, Xia W, Burris H 3rd, et al. 2005. Study of the biologic effects of lapatinib, a reversible inhibitor of ErbB1 and ErbB2 tyrosine kinases, on tumor growth and survival pathways in patients with advanced malignancies. J Clin Oncol, 23:2502-12.

Spector NL, Yarden Y, Smith B, et al. 2007. Activation of AMP-activated protein kinase by human EGF receptor 2/EGF receptor tyrosine kinase inhibitor protects cardiac cells. Proc Natl Acad Sci U S A, 104:10607-12.

Storniolo AM, Pegram MD, Overmoyer B, et al. 2008. Phase I dose escalation and pharmacokinetic study of lapatinib in combination with trastuzumab in patients with advanced ErbB2-positive breast cancer. J Clin Oncol, 26:3317-23.

Sunaga N, Tomizawa Y, Yanagitani N, et al. 2007. Phase II prospective study of the efficacy of gefitinib for the treatment of stage III/IV non-small cell lung cancer with EGFR mutations, irrespective of previous chemotherapy. Lung Cancer, 56:383-9.

Sweetman R, Lacouture ME, Koehler M, et al. 2007. Skin events (SE) among 1,126 patients (Pts) treated with lapatinib (L), an oral dual Erb1/2 tyrosine kinase inhibitor (TKI) [abstract]. J Clin Oncol, 25(18S): abstr 9102 .

Toi M, Iwata H, Fujiwara Y, et al. 2007. Biomarker analyses in Japanese refractory advanced breast cancer patients treated with lapatinib monotherapy [abstract]. San Antonio Breast Cancer Symposium, abstr 5114.

Toi M, Tominaga T, Osaki A, et al. 1994. Role of epidermal growth factor receptor expression in primary breast cancer: results of a biochemical study and an immunocytochemical study. Breast Cancer Res Treat, 29:51-8.

Vazquez-Martin A, Oliveras-Ferraros C, Colomer R, et al. 2008. Low-scale phosphoproteome analyses identify the mTOR effector p70 S6 kinase 1 as a specific biomarker of the dual-HER1/HER2 tyrosine kinase inhibitor lapatinib (Tykerb) in human breast carcinoma cells. Ann Oncol, 19:1097-109. 
Vogel CL, Cobleigh MA, Tripathy D, et al. 2002. Efficacy and safety of trastuzumab as a single agent in first-line treatment of HER2-overexpressing metastatic breast cancer. J Clin Oncol, 20:719-26.

von Minckwitz G, Jonat W, Fasching P, et al. 2005. A multicentre phase II study on gefitinib in taxane- and anthracycline-pretreated metastatic breast cancer. Breast Cancer Res Treat, 89:165-72.

Wood ER, Truesdale AT, McDonald OB, et al. 2004. A unique structure for epidermal growth factor receptor bound to GW572016 (Lapatinib): relationships among protein conformation, inhibitor off-rate, and receptor activity in tumor cells. Cancer Res, 64:6652-9.

Xia W, Bacus S, Hegde P, et al. 2006. A model of acquired autoresistance to a potent ErbB2 tyrosine kinase inhibitor and a therapeutic strategy to prevent its onset in breast cancer. Proc Natl Acad Sci U S A, 103:7795-800.

Xia W, Gerard CM, Liu L, et al. 2005. Combining lapatinib (GW572016), a small molecule inhibitor of ErbB1 and ErbB2 tyrosine kinases, with therapeutic anti-ErbB2 antibodies enhances apoptosis of ErbB2-overexpressing breast cancer cells. Oncogene, 24:6213-21.
Xia W, Husain I, Liu L, et al. 2007. Lapatinib antitumor activity is not dependent upon phosphatase and tensin homologue deleted on chromosome 10 in ErbB2-overexpressing breast cancers. Cancer Res, 67:1170-5.

Xia W, Mullin RJ, Keith BR, et al. 2002. Anti-tumor activity of GW572016: a dual tyrosine kinase inhibitor blocks EGF activation of EGFR/erbB2 and downstream Erk1/2 and AKT pathways. Oncogene, 21:6255-63.

Yarden Y, Sliwkowski MX. 2001. Untangling the ErbB signalling network Nat Rev Mol Cell Biol, 2:127-37.

Zhou X, Segreti A, Cameron D, et al. 2008. Effect of lapatinib plus capecitabine $(\mathrm{L}+\mathrm{C})$ on quality of life $(\mathrm{QOL})$ compared to capecitabine (C) alone ine ErbB+ metastatic breast cancer (MBC): an exploratoy analysis [abstract]. J Clin Oncol, 26(Suppl):abstr 9532.

Zhou Y, Li S, Hu YP, et al. 2006. Blockade of EGFR and ErbB2 by the novel dual EGFR and ErbB2 tyrosine kinase inhibitor GW572016 sensitizes human colon carcinoma GEO cells to apoptosis. Cancer Res, 66:404-11. 